\title{
Predicting binary choices from probability phrase meanings
}

\author{
Thomas S. Wallsten \\ University of Maryland, College Park, Maryland \\ AND \\ YOONHEE JANG \\ University of California, San Diego, La Jolla, California
}

\begin{abstract}
The issues of how individuals decide which of two events is more likely and of how they understand probability phrases both involve judging relative likelihoods. In this study, we investigated whether derived scales representing probability phrase meanings could be used within a choice model to predict independently observed binary choices. If they can, this simultaneously provides support for our model and suggests that the phrase meanings are measured meaningfully. The model assumes that, when deciding which of two events is more likely, judges take a single sample from memory regarding each event and respond accordingly. The model predicts choice probabilities by using the scaled meanings of individually selected probability phrases as proxies for confidence distributions associated with sampling from memory. Predictions are sustained for 34 of 41 participants but, nevertheless, are biased slightly low. Sequential sampling models improve the fit. The results have both theoretical and applied implications.
\end{abstract}

In this article, we bring together two theoretical issues that should have been united long ago. One of them concerns how individuals decide which of two events or answers to a question is more likely to occur or to be correct; the other concerns how individuals understand probability phrases (e.g., unlikely, almost certain). The issues overlap because they both involve judging relative likelihoods and, therefore, at least in part, invoke common underlying cognitive processes. We concentrate on natural language rather than on numerical descriptions of event likelihood, because that is the mode that people overwhelmingly prefer when reporting their internal or epistemic uncertainty (e.g., Brun \& Teigen, 1988; Erev \& Cohen, 1990; Olson \& Budescu, 1997).

Despite the considerable research on how people understand the natural language of uncertainty, there is no well-accepted and theoretically validated way to measure the subjective meanings of probability phrases. Recently, Dhami and Wallsten (2005) introduced a new measurement technique that yields a representation of phrase meanings as second-order probability distributions (i.e., distributions of probabilities), which they termed probability signatures. Lacking in their article, however, was a theoretical structure within which to validate the representations. In this article, we fill that void, developing and reporting our test of a new stochastic-choice model that uses the probability signatures to predict individuals' relative likelihood judgments regarding pairs of uncertain events. The results are of both theoretical and applied interest.. At the theoretical level, they constitute an advance in our knowledge of the relationship between meaning and action. At the applied level, they provide a framework for improving communication between expert and decision maker.

We first briefly and critically review the literature on quantifying the meanings of probability phrases, then summarize relevant aspects of binary-choice models, and finally develop a theoretical structure that uses phraseprobability signatures to predict independent-choice probabilities regarding which of two events is more likely to occur or to be true. The experiment and discussion follow.

\section{Quantifying Probability Phrases}

Two findings about how individuals use and interpret probability phrases are clear. First, people have vastly different probability lexicons (see, e.g., Budescu, Weinberg, \& Wallsten, 1988; Dhami \& Wallsten, 2005; Erev \& Cohen, 1990; Karelitz \& Budescu, 2004). For example, in Dhami and Wallsten, 29 respondents, who were asked to create lexicons containing 7 terms, generated 102 distinct probability phrases, of which only 38 appeared in two or more lexicons. The second well-documented result is the large between-subjects variability in converting probability phrases to numbers (see, e.g., Beyth-Marom, 1982; Budescu \& Wallsten, 1985; Lichtenstein \& Newman, 1967; Simpson, 1963). Additionally, as Wallsten, Budescu,

T. S.Wallsten, twallsten@psyc.umd.edu 
Rapoport, Zwick, and Forsyth (1986) pointed out, individuals understand probability terms as imprecise, rather than precise, quantifications of uncertainty. Wallsten et al. suggested that phrase meaning might be represented more accurately in terms of membership functions (MFs) over the $[0,1]$ probability interval. A phrase's MF assigns to each probability a real number that represents the degree of the phrase's membership in the concept defined by the phrase. Budescu and Wallsten (1995) summarized much of the earlier literature; more recent studies have been summarized by Karelitz and Budescu, who used MFs to translate across individuals' lexicons.

However, there are two problems with the MF methodology. One, acknowledged in many of the MF articles, is that there is no natural way to associate an error theory with MFs, rendering statistical comparisons tenuous. The other, more subtle problem concerns validation. The validation methods to date have relied on using MFs to predict one set of phrase-probability associations from another rather than to predict entirely different choices - for example, regarding which of two events is judged to be more likely. The present research addressed both issues.

\section{Probability Signatures}

Since our model depends on representing phrase meanings in terms of probability signatures, we first summarize Dhami and Wallsten's (2005) methodology and results. Their participants created seven probability phrases to populate their lexicons and ranked the phrases. Then, they made judgments that yielded probability signatures. ${ }^{1}$ The participants saw 101 circles, each divided into two radial sectors, such that the red sector covered $0 \%, 1 \%, \ldots, 99 \%, 100 \%$ of the area and the black sector covered the rest. For each circle, the participants selected one phrase in their lexicon that best described the probability that a randomly spun pointer would land on the red sector. As a consequence, each phrase was associated with a distribution of probabilities - the phrase's probability signature. The signatures for a given respondent were well fit by equal-variance normal distributions over the probabilities, $p$, converted to $\log$ odds, $\log [p /(1-p)]$. Figure 1 shows a family of (unequal-variance) probability signatures for a single participant in the experiment reported below. Of particular interest in Dhami and Wallsten, signatures for equally ranked phrases were more similar to each other across respondents than were signatures for common phrases at different ranks. This result is consistent with Karelitz and Budescu's (2004), in that probability phrases were best translated across lexicons via rank orders.

Dhami and Wallsten (2005) pointed out that probability signatures based on associating phrases with spinner probabilities are an attractive way to scale phrase meanings because, unlike scaling in other psychophysical domains, the results are comparable across individuals. This is so because two conditions hold. One is that the scale is bounded by the universally understood endpoints of $p=0$, representing impossibility, and $p=1$, representing certainty, creating a common unit for all judges. The other is that "when the axioms leading to a probability measure are satisfied, the resulting scale is unique ... and thus comparable from one person to the next" (Dhami \& Wallsten, 2005, p. 1058). The authors argued that the axioms do hold when individuals judge clear generic events, such as binary spinners. However, despite the scales' comparability across individuals, they are still not validated in a process-oriented theoretical sense, because they have not been shown to predict independent behaviors on the part of individuals. We now briefly review choice models and then develop one within which such prediction can take place.

\section{Choice Models}

It is reasonable to assume that when judging which of two events (from the same or different domains) is more likely, one searches memory and retrieves and weighs arguments and evidence for and against each event, which causes opinion regarding each event to vary in strength and direction, until eventually one decides that one event is more likely than the other to be true and indicates a choice.

This suggestion is only a minor variation on others in the literature. Koriat, Lichtenstein, and Fischhoff (1980) argued that when people judge the likelihoods of individual events, they first search their knowledge base (in a biased manner, as the authors claimed) and subsequently evaluate the evidence. The availability and representativeness heuristics, which also focus on judging individual events, rely on memory search and related evaluations (see, e.g., Tversky \& Kahneman, 1974). Perhaps the most carefully worked out model relating memory search to judging individual events is MINERVA-DM (Dougherty, Gettys, \& Ogden, 1999).

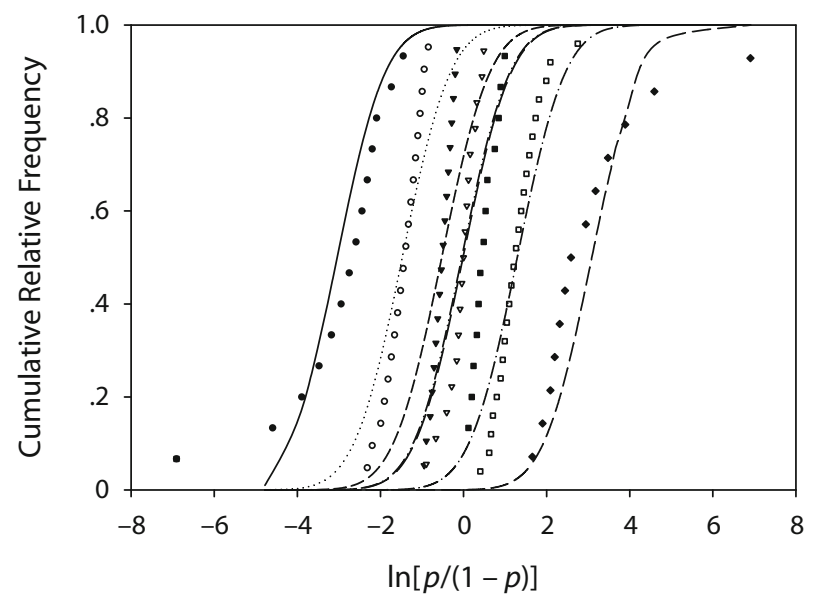

Figure 1. A family of probability signatures for a single participant. This person's lexicon consisted of the following phrases in order from lowest to highest probability signature (left to right on the graph), highly unlikely, not likely, somewhat possible, possibly, probably, good chance, and definitely. Points show the empirical cumulative distribution and curves show the theoretical distributions obtained by fitting the data with unequal-variance normal distributions over probabilities, $p$, converted to $\log$-odds, $\log [p /$ $(1-p)]$. Converting back to $p$, the theoretical means of the distributions are $\hat{p}_{1}=.06, \hat{p}_{2}=.20, \hat{p}_{3}=.36, \hat{p}_{4}=.51, \hat{p}_{5}=.64, \hat{p}_{6}=$ .80 , and $\hat{p}_{7}=.95$. 
If we turn to models of pair-comparison judgments, Wallsten and González-Vallejo's (1994) stochastic judgment model added a third stage to Koriat et al.'s (1980) model, resulting in the three stages of (1) memory search, (2) evaluation, and (3) response. The "fast and frugal" heuristics discussed by Gigerenzer and his colleagues also rely on memory search, although in a very different fashion from that which is envisioned in other models (e.g., Gigerenzer \& Todd, 1999; Goldstein \& Gigerenzer, 2002). However, the models that are closest in spirit to the memory search and evaluation processes envisioned here are the sequential-sampling models, applied to speeded-choice and discrimination tasks (e.g., Diederich \& Busemeyer, 2006; Laming, 1968; Link \& Heath, 1975; Ratcliff \& Rouder, 1998), as well as to choice and decision tasks of a more deliberative nature (e.g., Busemeyer \& Townsend, 1993). All of these models involve a process that drifts to one decision boundary or to the other as information accumulates. When the process crosses a boundary, the individual's decision is made. Recorded dependent variables are decision time and the actual choice.

\section{Predicting Binary Choices \\ From Probability Signatures}

Our goal is for our model to predict each respondent's binary choice probabilities from his or her probability signatures. This aim differs from those for all other models and requires its own developments. Participants engage in four tasks in our paradigm: (1) develop their lexicons, (2) establish probability signatures for their selected phrases, (3) use those phrases to describe their confidence that well-defined events will be true or will occur within a fixed time interval, and (4) view the events again, now in pairs, and indicate which one is more likely to be true.

The model of choice-probability prediction from probability signatures is simple but differs in a fundamental way from others. It is based on the assumption that when judging the likelihood that an event is true or will occur, if one is not certain of the answer, one sequentially searches memory for evidence or related information, which causes opinion to fluctuate over time. This fluctuation results in a distribution of degrees of confidence, experienced phenomenologically as imprecise opinion, which we model as a second-order probability distribution. This distribution underlies both probability phrase assignment and paircomparison judgment.

Consider binary choice first. For simplicity, assume that when asked to decide whether event $A$ or $B$ is more likely true, one takes a single sample from memory regarding each event and chooses according to which one yields the greater confidence. Subsequently, we consider plausible sequential-sampling schemes, of which this is a special case. For a single pair of samples, the probability that $A$ is chosen rather than $B$ is the probability that the sample from the second-order distribution for $A$ is greater than that for $B, P(A>B)$. Specifically, if we let $G_{i}(p)$ and $g_{i}(p)$ refer to the second-order cumulative and probability mass distribution functions, respectively, for events
$i=(A, B)$, the probability that forecast $A$ is chosen over forecast $B$ is

$$
P(A \succ B)=\int_{0}^{1} G_{B}(p) \cdot g_{A}(p) d p .
$$

Now consider the assignment of probability phrases to events. An individual's lexicon consists of phrases, $W_{1}$, $W_{2}, \ldots, W_{n}$, each of which has an imprecise meaning for him or her, which we have scaled as the probability signature. We assume that when one is required to use a linguistic phrase to represent one's judgment about the likelihood of an event, one selects the phrase whose meaning most closely matches his or her imprecise opinion of the event's likelihood. In terms of the model, we assume that the judge selects the phrase whose probability signature most closely matches the event's second-order probability distribution.

Let $F_{i}$ and $f_{i}$ denote the cumulative and probability mass distribution functions, respectively, representing the probability signature of phrase $W_{i}$ for $i=1,2, \ldots, n$. If the judge has assigned phrase $W_{i}$ to forecast $A$ and $W_{j}$ to forecast $B(i \neq j)$, then a good estimate of $P(A>B)$ in Equation 1 is

$$
\hat{P}(A \succ B)=\int_{0}^{1} F_{j}(p) \cdot f_{i}(p) d p .
$$

The experiment that follows simultaneously tested the choice model and the method of scaling probability signatures by comparing empirical estimates of the choice probabilities in Equation 1 to the values independently predicted via the probability signatures in Equation 2.

\section{METHOD}

\section{Participants}

Forty-one undergraduate students at the University of Maryland at College Park volunteered to participate in this experiment for course credit.

\section{Materials and Procedure}

The experiment consisted of four tasks and lasted approximately 50 min.

Task 1. We provided participants with lists of 21 modifiers (e.g., very, not) and 18 probability adjectives (e.g., likely, possible) to aid them in creating lexicons of 5-12. They constructed probability phrases by clicking on an adjective and zero, one, or two modifiers (e.g., likely, very likely, not very likely). In addition, they were free to type in any phrases they wished. Upon completing their lexicons, they rank ordered the phrases from smallest to greatest likelihood.

Task 2. Next, participants encoded probability signatures for each phrase: The computer presented a wheel (spinner) divided into red and black radial areas along with the ordered lexicon and the instructions, "Check the phrase(s) that describe your confidence that the spinner will land on black." For each of 101 trials, consisting in random order of different proportions of black equally spaced from 0 to 1 , participants selected all the phrases that they considered to be appropriate descriptors.

Task 3. Next, participants saw a sequence of 100 forecasts of possible real-world events regarding current affairs, politics, entertainment, sports, and science, created to span the range from virtually impossible to virtually certain to occur, along with their ordered list of phrases. For each event, they selected the single phrase that best 
Table 1

The Number of Respondents and the Number of Rank Positions at Which Two Respondents Used the Same Phrase As a Function of Lexicon Size

\begin{tabular}{lllllllll}
\hline & \multicolumn{8}{c}{ Lexicon Size } \\
\cline { 2 - 9 } & 5 & 6 & 7 & 8 & 9 & 10 & 11 & 12 \\
\hline $\begin{array}{l}\text { Number of respondents } \\
\text { Number of rank }\end{array}$ & 4 & 5 & 10 & 4 & 4 & 3 & 3 & 8 \\
$\begin{array}{l}\text { positions at which } \\
\text { two respondents used } \\
\text { the same phrase }\end{array}$ & 1 & 0 & 6 & 1 & 2 & 0 & 1 & 2 \\
\hline
\end{tabular}

described their confidence that it would occur. The order of events was randomized for each participant.

Task 4. Finally, the computer sequentially presented 40 pairs of events, one above the other on the screen, selected from those judged in Task 3. On each trial, the participants chose the event they considered more likely to occur. To control the choice difficulty, the computer rank ordered the events according to the phrases assigned to them and created four types of pairs on the basis of differences in the event rankings. The most difficult pairs contained events that differed by one rank position - that is, they were at ranks $r$ and $r+1$; the next most difficult pairs had events at ranks $r$ and $r+2$, then at ranks $r$ and $r+3$, and finally at ranks $r$ and $r+4$. There were 10 pairs at each level, and the 40 were presented in a random order for each participant.

\section{RESULTS}

\section{Individual Probability Phrases}

Table 1 shows the number of respondents selecting each lexicon size and, within lexicon size, the number of rank positions at which two people used the same phrase. For example, two out of four people with a lexicon comprising five phrases used a common phrase at one rank position. All but 1 of the 41 participants used modifiers in selecting phrases. In total, the respondents created 170 distinct phrases, of which 98 appeared in a single lexicon and 72 appeared in two or more lexicons. The median lexicon size across respondents was $8(M=8.46, S D=2.39)$.
Table 2 provides some details on the 14 phrases that appeared in five or more lexicons, including the sizes of the lexicon and the ranks within lexicons at which the phrases appeared. ${ }^{2}$ For example, of the 15 participants who used good chance, one with a lexicon comprising five phrases ranked it 2 , one with a lexicon comprising six phrases ranked it 4 and another ranked it 5, and so on. Table 2 also shows a few inconsistencies - for example, a ranking of 2 for absolutely certain by a participant with a lexicon comprising 11 phrases, and a ranking of 11 for no chance by a participant with a lexicon comprising 12 phrases. In fact, three participants ranked the phrases inconsistently. ${ }^{3}$

\section{Fitting Distributions to the Probability Phrase}

Turning to the probability signatures, the mean number of probability values (out of 101) that participants associated with each phrase in Task 2 was $17.42(S D=5.36$; $M d n=17.39$ ). Following Dhami and Wallsten (2005), we estimated a normal on log-odds distribution over the empirical distribution of probability values for each phrase within individuals, but unlike them, we did not constrain the variances to be equal. That is, we transformed the probability values to log odds, calculated the mean and variance per phrase per individual, and used those statistics as the distribution parameters. ${ }^{4}$ Figure 1 shows the empirical and theoretical distributions for a single participant with a lexicon comprising seven phrases, plotted as cumulative distributions over log odds. For each participant, we used the Kolmogorov-Smirnov test to determine whether the fitted and empirical distributions differed significantly. ${ }^{5}$ There were no significant differences between the two distributions for 33 out of 41 participants $(80.5 \%)$.

\section{Prediction of Choice Probability}

For any pair of forecasts, let $A$ denote the one associated with the higher ranked phrase and $B$ the one associated with the lower ranked phrase. The observed proportion of consistent choices was calculated for each participant as the proportion of times he or she selected forecast

Table 2

The Total Number of Respondents Using Each Phrase and the Ranks at Which They Appeared As a Function of Lexicon Size for Total Numbers $\geq 5$

\begin{tabular}{|c|c|c|c|c|c|c|c|c|c|}
\hline \multirow[b]{2}{*}{ Phrase } & \multirow{2}{*}{$\begin{array}{c}\text { Total } \\
\text { Number }\end{array}$} & \multicolumn{8}{|c|}{ Lexicon Size } \\
\hline & & 5 & 6 & 7 & 8 & 9 & 10 & 11 & 12 \\
\hline Good chance & 15 & 2 & 4,5 & $\begin{array}{l}4,4,5 \\
5,6,6\end{array}$ & 5 & $6,7,9$ & & 11 & 9 \\
\hline Very likely & 9 & 3 & & 5 & 7 & 8 & 6 & 7 & $8,11,12$ \\
\hline Absolutely certain & 8 & & 6,6 & 7 & 8 & 10 & & 2,11 & 12 \\
\hline Highly unlikely & 8 & 1 & 1 & 1,3 & & 2,8 & 2 & & 3 \\
\hline Almost certain & 7 & & 3,6 & 5 & 8,8 & 3,8 & & & \\
\hline Most likely & 7 & 4 & & 6 & 7 & & 10 & 9 & 9,10 \\
\hline No chance & 7 & & & 2 & 1 & 1,1 & & 1 & 2,11 \\
\hline Probably & 7 & & & 4,5 & 2 & 6 & & & $8,11,12$ \\
\hline Not likely & 6 & 1 & 2 & $2,3,3$ & & & & 8 & \\
\hline Very possible & 6 & 4 & & 5 & 4 & 7 & & 9 & 11 \\
\hline Very unlikely & 6 & & & 2 & 2,3 & & 3 & 5 & 2 \\
\hline Impossible & 5 & & & 1,1 & 1 & & & & 1,1 \\
\hline Not possible & 5 & & & 1 & & & 1 & & $1,2,10$ \\
\hline Pretty unlikely & 5 & 3,3 & & 2 & & 3,3 & & & \\
\hline
\end{tabular}


$A$ over $B$. Equation 2 provides the means for calculating the corresponding predicted probability for any particular pair. In terms of log odds, Equation 2 is reexpressed as

$$
P_{i}(A \succ B)=\int_{-\infty}^{1-\infty} F_{B}(x) \cdot f_{A}(x) d x,
$$

where $x=\log [p /(1-p)]$ and $i$ is a number from 1 to 40 denoting the pair. The predicted proportion of choices of $A$ over $B$ is the simple average of the theoretical choice probabilities over the 40 pairs. Note that the predictions do not entail fitting any parameters. Figure 2 shows the observed versus predicted proportion of consistent choices over the 40 pairs; each point represents a single individual. We used a chi-square test with $d f=1$ to compare observed with predicted choice probabilities at the individual level. The test failed for 7 of the 41 participants, all of whom fall within the oval in Figure 2.6 To increase power, we summed the chi-squares for an overall test with $d f=41$. The model failed this test $\left[\chi^{2}(41)=248.42\right]$. Note, however, that the empirical choice probabilities of the seven individuals within the oval were all at or below chance. If they are excluded from the group analysis, then the group test yields $\chi^{2}(34)=42.08(p=.161)$ and the model does not fail. Despite the excellent collective fit for these 34 participants, note that the deviations from the diagonal were systematic; in most cases the empirical choice probabilities exceeded the predicted probabilities by small amounts. We modify the model to address this point in the Discussion section.

Figure 3 provides a more detailed view of the model fit at the individual level by plotting observed versus predicted choice probabilities for each participant, with a separate data point for each level of pair difficulty. The first 29 panels show the participants (represented by filled diamonds in Figure 2) for whom the theoretical values well describe both the empirical probability signatures and the mean choice probability. The next 5 panels (open circles in Figure 2) show the participants for whom the mean choice probabilities were well predicted despite poorly fitting probability signatures. The last 7 panels show the participants contained within the oval of Figure 2. Of those, the first 4 show participants (solid squares in Figure 2) whose probability signatures fit well, but for whom the choice model failed. Finally, the last 3 panels show the participants (stars in Figure 2; mentioned in note 3) for whom the probability signatures were poorly described and for whom the choice model failed. Individual chi-square tests with $d f=4$ comparing observed with predicted choice probabilities over the four difficulty levels show that the model fails for the same 7 participants as in Figure 2 and not for the others. When the chi-squares are summed, the model fails for the group as a whole $\left[\chi^{2}(164)=310.2\right]$. However, the model is not rejected when the 7 randomly responding participants are excluded $\left[\chi^{2}(136)=102.5\right.$, $p=.986]$.

\section{DISCUSSION}

For 34 of 41 participants, without fitting any free parameters the probability signatures predicted well both the

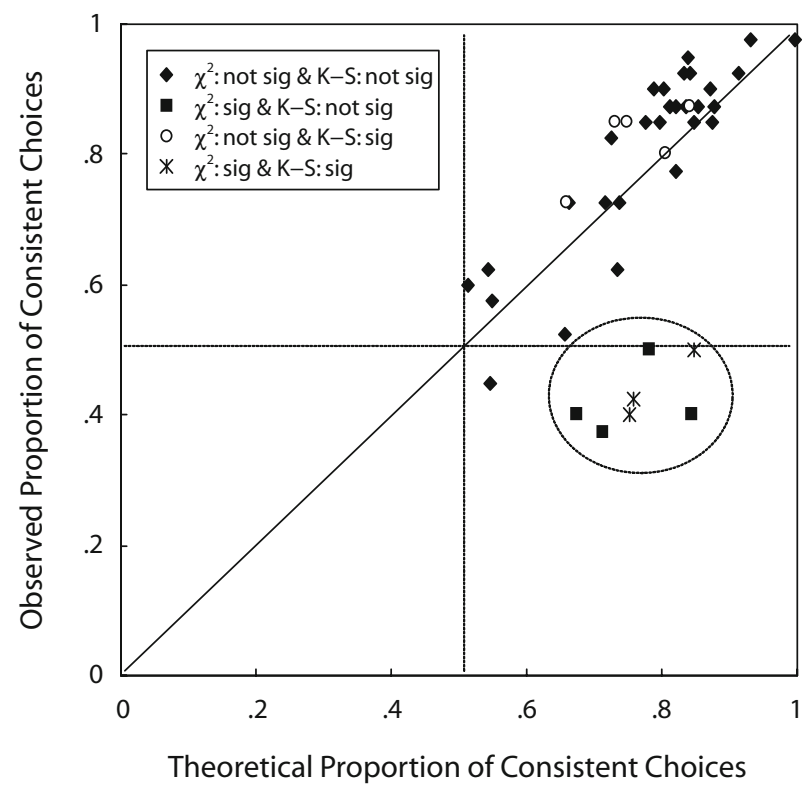

Figure 2. Observed versus theoretical proportion of consistent choices. The 29 filled-diamond data points were not significant on either the chi-square test of prediction of choice probabilities or the Kolmogorov-Smirnov (K-S) test of fit of normal to probability signature. The five open-circle data points were not significant on the chi-square test of prediction of choice probabilities but were significant on the $\mathrm{K}-\mathrm{S}$ test of fit of normal to probability signature. The four filled-square data points were significant on the chi-square test of prediction of choice probabilities but were not significant on the $\mathrm{K}-\mathrm{S}$ test of fit of normal to probability signature. The three star data points were significant on both the chisquare test of prediction of choice probabilities and the $\mathrm{K}-\mathrm{S}$ test of fit of normal to probability signature. The seven data points within the oval (i.e., the four data points of filled squares and the three data points of stars) are ones for which the model failed to predict the observed proportion. Sig = significant.

mean choice probabilities (Figure 2 ) and the choice probability pattern as a function of difficulty (Figure 3 ). This result is not a consequence of low-powered tests, because it holds for these 34 individuals at the group level as well. Nevertheless, it is apparent from Figure 2 that the theoretical values systematically underpredicted the empirical values, and therefore the model can be improved.

Recall that according to the model, when choosing whether event $A$ or $B$ is more likely, individuals respond after sampling memory once for each event. Relaxing this restriction to provide for sequential sampling allows the model to come closer to the data. One variation of sequential sampling assumes that individuals take a single memory sample regarding each event and make a choice if the difference in confidence between the two samples exceeds a threshold level, $c$. Otherwise they take another pair of samples and respond if the confidence difference exceeds $c$. Successive samples are not cumulated. The process continues until a choice is made.

To formalize this idea, let $p_{i}$, where $i=(A, B)$, denote the confidence associated with a single memory sample for the indicated event. The two assumptions are that (1) the respondent sets a criterion such that he or she chooses event $A$ if $p_{A}-p_{B}>c$, chooses event $B$ if $p_{B}-p_{A}>c$, and 

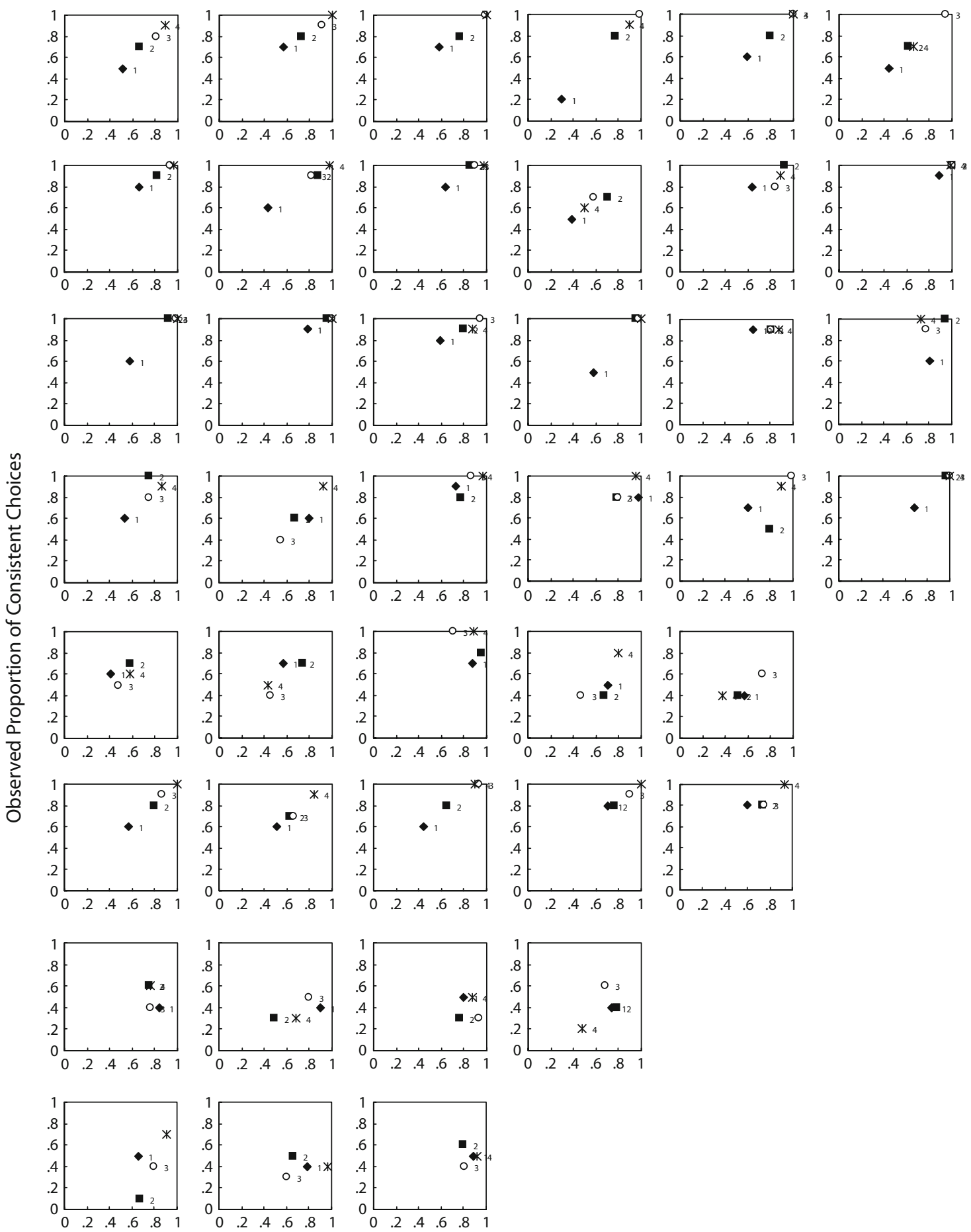

Theoretical Proportion of Consistent Choices

Figure 3. Scatterplots of the observed proportion of consistent choices against the theoretical proportion of consistent choices for each of the four types of pairs within each individual. Each number (i.e., 1, 2,3, or 4) indicates the difference between the rank orders of the phrases that were assigned to the two members of the pair; that is, $1=$ one rank order between the phrases, which indicates the most difficult choice task. The first 29 plots come from those whose data were not significant on either the chi-square test of prediction of choice probabilities or the Kolmogorov-Smirnov (K-S) test of fit of normal to probability signature. The next five plots come from those whose data were not significant on the chi-square test of prediction of choice probabilities but were significant on the $\mathrm{K}-\mathrm{S}$ test of fit of normal to probability signature. The next four plots come from those whose data were significant on the chi-square test of prediction of choice probabilities but were not significant on the $\mathrm{K}-\mathrm{S}$ test of fit of normal to probability signature. The final three plots come from those whose data were significant on both the chi-square test of prediction of choice probabilities and the $\mathrm{K}-\mathrm{S}$ test of fit of normal to probability signature. The data of the last seven plots failed to predict the observed proportion of consistent choices. 
otherwise takes another sample; and (2) sample impact is not cumulated. Let $q=P\left(p_{A}-p_{B}>c\right)$ for a single pair of samples and $s=P\left(-c<p_{A}-p_{B}<c\right)$ - that is, the probability that the sample does not exceed the threshold and another sample pair is drawn. ${ }^{7}$ It is easy to show in this case that

$$
P(A \succ B)=\frac{q}{1-s} \text { and } P(B \succ A)=\frac{1-q-s}{1-s} .
$$

The single free parameter in this model is $c$. When $c=0$, we are back to the single-sample model of Equation 1 [because $q$ is the value of $P(A>B)$ in Equation 1]. However, when $c>0,1-s<1$, and therefore

$$
\frac{q}{1-s}>q \text {. }
$$

Thus, for $c>0$, the predicted choice probability of this sequential noncumulative model (Equation 3) always exceeds that of the original single-sample version (Equation 1), as is required to improve the fit of the data points above the diagonal in Figure 2.

Sequential sampling models that assume cumulative impact of successive models will also yield more extreme predictions of $P(A>B)$ than Equation 1 does. We leave such developments for future research.

Focusing now on the statistically good fit instead of on deviations from it, the results provide simultaneous support to our simple choice model (or improvements thereof) and to the claim that we have measured the meanings of the probability phrases to individuals. The results are of both theoretical and methodological interest, which we consider in turn.

In terms of theory, having already discussed how the choice model may be improved, we turn to the measurement of phrase meaning. Recall that the results are at the level of individual self-selected phrases. Normal over logodds distributions provided excellent fits to the empirical second-order probability relative frequencies. It should be noted that although symmetric in log-odds space, when converted back to distributions over probabilities, these distributions are symmetric only when the mean is .5 and skew increasingly inward as the mean departs from .5 in either direction - as one would expect for any representation of phrase meaning. Our results differ from Dhami and Wallsten's (2005) slightly, in that they fit equal-variance normal on log-odds distributions to each respondent's set of seven probability signatures, whereas we did not constrain the variances to be equal. When we did so constrain them, the resulting functions did not predict the choice probabilities as well; we conclude that the unequalvariance case is the better representation.

At a methodological or applied level, probability signatures have a level of construct validity not established for other scaling methods and they are unique in a measurement-theoretic sense. Therefore, they provide a useful tool to investigate theories of verbal information processing and to incorporate verbal probabilities in applied decision analysis, including the prospect of translating from one person's lexicon of uncertainty to another's.

\section{AUTHOR NOTE}

We thank Paul Hanges for useful discussions regarding the theoretical developments in the Discussion section. Correspondence should be addressed to T. S. Wallsten, Department of Psychology, University of Maryland, College Park, MD 20742 (e-mail: twallsten@psyc.umd .edu).

\section{REFERENCES}

Beyth-Marom, R. (1982). How probable is probable? A numerical translation of verbal probability expressions. Journal of Forecasting, 1, 257-269.

Brun, W., \& Teigen, K. H. (1988). Verbal probabilities: Ambiguous, context-dependent, or both? Organizational Behavior \& Human Decision Processes, 41, 390-404.

Budescu, D. V., \& Wallsten, T. S. (1985). Consistency in interpretation of probabilistic phrases. Organizational Behavior \& Human Decision Processes, 36, 391-405.

Budescu, D. V., \& Wallsten, T. S. (1995). Processing linguistic probabilities: General principles and empirical evidence. In D. L. Medin (Series Ed.) and J. R. Busemeyer \& R. Hastie (Vol. Eds.), The psychology of learning and motivation: Vol. 32. Decision making from the perspective of cognitive psychology (pp. 275-318). San Diego: Academic Press.

Budescu, D. V., Weinberg, S., \& Wallsten, T. S. (1988). Decisions based on numerically and verbally expressed uncertainties. Journal of Experimental Psychology: Human Perception \& Performance, 14, 281-294.

Busemeyer, J. R., \& Townsend, J. T. (1993). Decision field theory: A dynamic-cognitive approach to decision making in an uncertain environment. Psychological Review, 100, 432-459.

DhAmi, M. K., \& WAllsten, T. S. (2005). Interpersonal comparison of subjective probabilities: Toward translating linguistic probabilities. Memory \& Cognition, 33, 1057-1068.

Diederich, A., \& Busemeyer, J. R. (2006). Modeling the effects of payoff on response bias in a perceptual discrimination task: Boundchange, drift-rate-change, or two-stage-processing hypothesis. Perception \& Psychophysics, 68, 194-207.

Dougherty, M. R. P., Gettys, C. F., \& Ogden, E. E. (1999). MINERVA-DM: A memory processes model for judgments of likelihood. Psychological Review, 106, 180-209.

EREv, I., \& COHEN, B. L. (1990). Verbal versus numerical probabilities: Efficiency, biases, and the preference paradox. Organizational Behavior \& Human Decision Processes, 45, 1-18.

Gigerenzer, G., \& TodD, P. M. (1999). Simple heuristics that make us smart (Evolution and cognition). New York: Oxford University Press.

Goldstein, D. G., \& Gigerenzer, G. (2002). Models of ecological rationality: The recognition heuristic. Psychological Review, 109, $75-90$.

Karelitz, T. M., \& Budescu, D. V. (2004). You say "probable" and I say "likely": Improving interpersonal communication with verbal probability phrases. Journal of Experimental Psychology: Applied, 10, 25-41.

Koriat, A., Lichtenstein, S., \& Fischhoff, B. (1980). Reasons for confidence. Journal of Experimental Psychology: Human Learning \& Memory, 6, 107-118

LAMING, D. R. J. (1968). Information theory of choice-reaction times. New York: Academic Press.

Lichtenstein, S., \& Newman, J. R. (1967). Empirical scaling of common verbal phrases associated with numerical probabilities. Psychonomic Science, 9, 563-564.

Link, S. W., \& Heath, R. A. (1975). A sequential theory of psychological discrimination. Psychometrika, 40, 77-105.

Olson, M. J., \& Budescu, D. V. (1997). Patterns of preference for numerical and verbal probabilities. Journal of Behavioral Decision Making, 10, 117-131.

Ratcliff, R., \& Rouder, J. N. (1998). Modeling response times for two-choice decisions. Psychological Science, 9, 347-356.

Simpson, R. H. (1963). Stability in meanings for quantitative terms: A 
comparison of meaning over 20 years. Quarterly Journal of Speech, 49, 146-151.

TVersky, A., \& Kahneman, D. (1974). Judgment under uncertainty: Heuristics and biases. Science, 185, 1124-1131.

Wallsten, T. S., Budescu, D. V., Rapoport, A., Zwick, R., \& ForSYTH, B. (1986). Measuring the vague meanings of probability terms. Journal of Experimental Psychology: General, 115, 348-365.

Wallsten, T. S., \& GonZÁlez-Vallejo, C. (1994). Statement verification: A stochastic model of judgment and response. Psychological Review, 101, 490-504.

\section{NOTES}

1. Dhami and Wallsten (2005) obtained four sets of judgments: two that yielded probability signatures and two that established MFs in the same way that Karelitz and Budescu (2004) did. We focus here on one of the probability signature methods.
2. Three participants ranked their lexicons in the opposite direction. We reversed the orders before analyzing the data.

3. These are different from the 3 participants who consistently ranked in the opposite direction. Careful analysis showed no consistent ordering in these individuals' rankings.

4. Values of $p$ equal to 0 and 1 were replaced by .001 and .999, respectively.

5. All differences that we report as statistically significant have $\alpha<$ .05 .

6 . These 7 participants include the 3 who ranked their phrases inconsistently, as well as 4 others.

7. For completeness, $P\left(p_{B}-p_{A}>c\right)=1-q-s$.

(Manuscript received October 21, 2007;

revision accepted for publication February 15, 2008.) 